\title{
Dynamic Similarity of Oscillatory Flows Induced by Nanomechanical Resonators
}

\author{
Elizabeth C. Bullard, ${ }^{1}$ Jianchang Li, ${ }^{2}$ Charles R. Lilley, ${ }^{3}$ Paul Mulvaney, ${ }^{2}$ Michael L. Roukes, ${ }^{1}$ and John E. Sader ${ }^{1,3, *}$ \\ ${ }^{1}$ Kavli Nanoscience Institute and Departments of Physics, Applied Physics, and Bioengineering, California Institute of Technology, \\ Pasadena, California 91125, USA \\ ${ }^{2}$ School of Chemistry and Bio21 Institute, The University of Melbourne, Victoria 3010, Australia \\ ${ }^{3}$ Department of Mathematics and Statistics, The University of Melbourne, Victoria 3010, Australia
}

(Received 3 July 2013; published 7 January 2014)

\begin{abstract}
Rarefied gas flows generated by resonating nanomechanical structures pose a significant challenge to theoretical analysis and physical interpretation. The inherent noncontinuum nature of such flows obviates the use of classical theories, such as the Navier-Stokes equations, requiring more sophisticated physical treatments for their characterization. In this Letter, we present a universal dynamic similarity theorem: The quality factor of a nanoscale mechanical resonator at gas pressure $P_{0}$ is $\alpha$ times that of a scaled-up microscale resonator at a reduced pressure $\alpha P_{0}$, where $\alpha$ is the ratio of nanoscale and microscale resonator sizes. This holds rigorously for any nanomechanical structure at all degrees of rarefaction, from continuum through to transition and free molecular flows. The theorem is demonstrated for a series of nanomechanical cantilever devices of different size, for which precise universal behavior is observed. This result is of significance for research aimed at probing the fundamental nature of rarefied gas flows and gas-structure interactions at nanometer length scales.
\end{abstract}

DOI: 10.1103/PhysRevLett.112.015501

PACS numbers: 81.70.-q, 62.25.-g, 81.07.-b

Miniaturization of resonant mechanical structures has driven progress across a wide range of advanced technologies, including sensors for mass detection and imaging with atomic resolution [1-3], monitoring of biological processes such as DNA hybridization [4], and mass spectrometry at the molecular scale [5]. While the constituent elastic properties of mechanical resonators remain identical to bulk values upon miniaturization to nanometer length scales [6-9], operation in fluid environments gives rise to physical phenomena not normally seen at macroscopic levels [10-14]. In liquid, these include transport due to the electrical nature of surfaces [15] and the possibility of violation of the usual no-slip condition [16]. Breach of no-slip is exacerbated for operation in gas, because the relevant molecular length scale is orders of magnitude larger than in liquid [17-19]. This leads to failure of the continuum hypothesis at relatively large length scales (microns), obviating the use of classical theories such as the Navier-Stokes equation.

Continuum theories are used ubiquitously in the design and characterization of macroscale and microscale mechanical structures. In the context of mechanical resonators in gas, this has provided insight into the fundamental physical processes underpinning their operation. For example, it is known that the quality factor (scaled inverse rate-ofenergy dissipation) of a mechanical resonator decreases as the size of the structure is reduced [10,20]. This is due to growth of the viscous penetration depth, where vorticity is prevalent, relative to the structure size. Yet this knowledge is predicated on the assumption that the gas mean free path $\lambda$ is much smaller than the size of the structure. The mean free path of air at $1 \mathrm{~atm}$ and room temperature is approximately $70 \mathrm{~nm}$ [21]. Thus, miniaturization of nanomechanical resonators to several hundred nanometers induces strong noncontinuum effects, which can deviate from the predictions of the Navier-Stokes equation.

The Knudsen number $\mathrm{Kn}$ is often used to characterize gas flow regimes and select appropriate theories for their calculation. This dimensionless parameter is defined as the ratio of the gas mean free path $\lambda$ to a characteristic length scale of the mechanical resonator $L_{0}$, i.e., $\mathrm{Kn} \equiv \lambda / L_{0}$. While analytical theories can often be formulated in the continuum $(\mathrm{Kn}<0.01)$, slip $(0.01<\mathrm{Kn}<0.1)$, and free molecular regimes $(\mathrm{Kn}>10)$ [22], flows in the transition regime demand consideration of the kinetic nature of the gas-this is precisely where many nanoscale resonators operate [22]. The Boltzmann equation dictates the behavior of a gas at arbitrary degrees of rarefaction, i.e., for all Knudsen numbers [17-19,22]

$$
\frac{\partial F}{\partial t}+c_{i} \frac{\partial F}{\partial x_{i}}+a_{i} \frac{\partial F}{\partial c_{i}}=\left[\frac{\partial F}{\partial t}\right]_{\text {coll }},
$$

where $F$ is the mass distribution function, $t$ is time, $x_{i}$ is position, $c_{i}$ is the molecular velocity, and $a_{i}$ is the body force per unit mass acting on the gas. The right-hand side of Eq. (1) is specified by the nature of intermolecular collisions, which generally results in a multidimensional phase space integral; for a hard sphere gas

$$
\left[\frac{\partial F}{\partial t}\right]_{\text {coll }}=\frac{d_{\mathrm{ref}}^{2}}{4 m} \int_{S^{2}} d \Omega \int_{R^{3}} d \boldsymbol{c}_{*}|\boldsymbol{g}|\left(F^{\prime} F_{*}^{\prime}-F F_{*}\right),
$$

where the subscript * refers to functions of incident molecular velocities, the prime denotes a function of postcollision velocities, $d_{\text {ref }}$ is the reference diameter of gas molecules, 
$\Omega$ is the solid angle, $\boldsymbol{g}$ is the relative pre- and post-collision molecular velocity, and $m$ the molecular mass. The complexity of the Boltzmann equation poses significant challenges to solution in comparison to classical continuum treatments that make use of the Navier-Stokes equations:

$$
\begin{aligned}
\frac{\partial \rho}{\partial t}+\frac{\partial}{\partial x_{i}}\left(\rho u_{i}\right)= & 0, \\
\rho\left(\frac{\partial u_{i}}{\partial t}+u_{j} \frac{\partial u_{i}}{\partial x_{j}}\right)= & -\frac{\partial P}{\partial x_{i}}+\mu \frac{\partial^{2} u_{i}}{\partial x_{j}^{2}} \\
& +\left(\mu_{B}+\frac{\mu}{3}\right) \frac{\partial^{2} u_{j}}{\partial x_{i} \partial x_{j}},
\end{aligned}
$$

for which numerical and asymptotic techniques are well advanced; $u_{i}$ is the mean velocity of the gas, $\rho$ is the gas density, $\mu$ the shear viscosity, $\mu_{B}$ the bulk viscosity, and $P$ is pressure. This situation is especially problematic for nanoscale resonators, which inherently operate in the transition regime-oscillatory flows generated by these structures require solution of the Boltzmann equation for rigorous analysis.

Advances in developing asymptotic and computational methods to solve the Boltzmann equation, particularly for low Mach number flows, have been reported recently [23-25]. These methods have been demonstrated for low-dimensional flows, such as Couette and Poiseuille flow. However, computational methods must be used for flows in the transition regime, which can be prohibitively expensive for practical nanoscale mechanical resonators with complicated geometries that produce genuinely three-dimensional time-dependent flows. Their use in predicting oscillatory flows generated by nanoscale resonators has been limited [26,27]. Characterization of gas-surface interactions and thus specification of appropriate boundary conditions can also be problematic, limiting the utility and accuracy of any such simulation $[17,18,22]$. Consequently, the characterization of gas flows at nanometer length scales poses a significant and open challenge in the physical sciences.

In this Letter, we report a general dynamic similarity theorem for accurate and routine evaluation of the quality factor of any nanoscale mechanical resonator in gas. This is derived rigorously using dimensional analysis [28] and obviates the need for sophisticated computational techniques of rarefied gas flow, which is currently required. It enables the use of microelectromechanical systems, for which highly precise fabrication methods are well established, in the design and characterization of nanoelectromechanical systems. This approach intrinsically accounts for all non-continuum processes, including the effects of gas rarefaction and gas-surface interactions. It also allows modeling of nanoscale mechanical resonators of arbitrary geometry and structure. The rate-of-energy dissipation in resonant mechanical structures dictates their sensitivities to external stimuli, and knowledge of this physical property is essential for the design and operation of ultrasensitive devices [29]. The dynamic similarity theorem presented here enables the straightforward characterization of this fundamental quantity.

Consider a linearly elastic mechanical structure, of arbitrary specifications, that is immersed in gas and undergoing resonant oscillations. Because of its linearity, the rate-ofenergy dissipation by the resonator depends on the square of its oscillation amplitude. Energy dissipation due to the gas also depends on the resonator size $L_{0}$, its radial resonant frequency in gas $\omega_{R}$, the gas shear viscosity $\mu$, gas density $\rho$, and mean free path, $\lambda$. The quality factor $Q$ of the resonator is related to its maximum energy stored $E_{\text {stored }}$, and energy dissipated per cycle $E_{\text {diss }}$, via $\left.Q \equiv 2 \pi\left(E_{\text {stored }} / E_{\text {diss }}\right)\right|_{\omega=\omega_{R}}$. Extending the dimensional analysis of Refs. [30,31] to include an extra physical quantity, the gas mean free path, yields the scaling law [32]

$$
k=\rho L_{0}^{3} \Omega(\operatorname{Re}, \mathrm{Kn}) \omega_{R}^{2} Q,
$$

where, $k$ is the resonator stiffness, $\Omega(\mathrm{Re}, \mathrm{Kn})$ is a dimensionless function that depends only on the Reynolds number [33], $\operatorname{Re} \equiv \rho L_{0}^{2} \omega_{R} / \mu$, and the Knudsen number, $\mathrm{Kn}=\lambda / L_{0}$; it also intrinsically depends on two other dimensionless groups: the mode shape of resonant oscillations and the geometry of the resonator. Note that viscosity is independent of the degree of gas rarefaction [17-19,30].

Next, we study the behavior of the Reynolds and Knudsen numbers as the resonator size and/or gas density and pressure are varied-all other properties of the resonator such as its material and geometry are held constant; this is well satisfied in practice [6-9]. The product of these dimensionless variables is

$$
\operatorname{Re} \operatorname{Kn}=\frac{(\rho \lambda)\left(\omega_{\mathrm{vac}} L_{0}\right)}{\mu} \frac{\omega_{R}}{\omega_{\mathrm{vac}}},
$$

where $\omega_{\mathrm{vac}}$ is the resonant frequency of the resonator in vacuum. The bracketed terms in Eq. (5) do not change as the gas density and/or resonator dimensions are uniformly scaled, i.e., increased or decreased [19]. Furthermore, the surrounding gas weakly affects the resonant frequency of a nanomechanical resonator, because the average density of the structure is orders of magnitude larger than the gas density [34]; equating these values is a good approximation. Equation (5) immediately establishes that the product $\mathrm{Re} \mathrm{Kn}$ is invariant, i.e., $\mathrm{Re}=a / \mathrm{Kn}$ where $a$ is a constant, as resonator size is uniformly scaled and/or gas pressure is varied [35], provided the gas used is the same. Therefore, the dimensionless function $\Omega(\mathrm{Re}, \mathrm{Kn})$ is only a function of the Knudsen number.

Equating the maximum potential and kinetic energies of the resonator gives $k=B \rho_{\text {av }} L_{0}^{3} \omega_{\text {vac }}^{2}$, where $\rho_{\text {av }}$ is its average density and $B$ is a dimensionless constant that depends 
on its geometry and mode shape. Combining this expression with Eq. (4) gives

$$
Q=\frac{\rho_{0}}{\rho}\left(\frac{\omega_{\mathrm{vac}}}{\omega_{R}}\right)^{2} H(\mathrm{Kn}),
$$

where the dimensionless function $H(\mathrm{Kn})$ is inversely proportional to $\Omega(a / \mathrm{Kn}, \mathrm{Kn}) \rho_{0} / \rho_{\mathrm{av}}$. The reference gas density $\rho_{0}$ can be chosen arbitrarily and simply defines the normalization of the dimensionless function $H(\mathrm{Kn})$; see discussion below. Again, approximating the resonant frequency in gas is by its value in vacuum (see above) leads to the required dynamic similarity theorem:

$$
Q=\frac{\rho_{0}}{\rho} H(\mathrm{Kn}) .
$$

Note that the Knudsen number Kn depends explicitly on the gas mean free path $\lambda$ and thus the gas density $\rho$.

Equation (7) is derived formally from dimensional considerations [28] and thus holds for any nanomechanical resonator, regardless of its geometry and mode shape. Once the dimensionless function $H(\mathrm{Kn})$ is determined for a particular resonator, the quality factor as the resonator size is uniformly scaled and/or the gas density adjusted, is immediately specified by Eq. (7). This is independent of the degree of gas rarefaction. We emphasize that this theorem applies to the quality factor due to the gas only; intrinsic dissipation within the resonator material is not considered.

Cantilever devices are chosen to assess the validity of this dynamic similarity theorem and are fabricated from 100, 300, and $500 \mathrm{~nm}$ thick SiN films deposited onto silicon wafers. Photolithography followed by a dry etch is used to define etch windows on the backside of the silicon wafers. $\mathrm{KOH}$ etching is used to create $\mathrm{SiN}$ membranes, which are then patterned with electron-beam lithography to define the cantilevers. A plasma etch is used to create the cantilevers, which are subsequently coated with gold for reflectivity. All devices are characterized by monitoring their Brownian fluctuations using optical interferometry over a range of gas pressures, from which the total quality factor $Q_{\text {total }}$ is measured. Dry nitrogen gas at room temperature is used in all measurements. The intrinsic quality factor $Q_{\text {int }}$ of each device is also measured (at very low pressures) and removed using the standard relation $1 / Q_{\text {total }}=1 / Q+1 / Q_{\text {int }}$ to yield the required quality factor due to the surrounding gas $Q$; only this gas quality factor is reported in the following figures, table, and discussion. Details of the experimental methodology are in the Supplemental Material [32].

Three sets of devices are fabricated with sizes uniformly scaled in the ratio $1: 3: 5$, in accord with the above thickness specifications, and include six different cantilever plan view geometries. This allows for thorough assessment of the dynamical similarity theorem. The upper row of Fig. 1 shows SEM micrographs of the triangular shaped device geometry (device $A$ ) at all three fabricated sizes - this illustrates the dimensional scaling of these devices. Importantly, the thicknesses of the silicon nitride and $\mathrm{Cr} / \mathrm{Au}$ coatings are uniformly scaled in synchrony with the plan view dimensions to ensure satisfaction of the underlying assumption of the dynamic similarity theorem; see Ref. [32]. SEM micrographs of the smallest versions of all other devices (devices $B, C, D, E$, and $F$ ) are given in the second and third rows of Fig. 1. The length scale $L_{0}$, chosen for the Knudsen number of each device is as follows. Devices $A, D, E, F$ : leg width at clamp; devices $B, C$ : width at free end.

We first consider device $E$, which corresponds to a cantilever resonator with a rectangular plan view and an aspect ratio (length to width) of 5. Importantly, analytical theories exist for the quality factor of this device in the continuum [10] and free molecular regimes [27,32,36,37]; the effect of varying the gas-surface interaction on the free molecular solution is presented in the Supplemental Material [32]. This allows for independent confirmation of the robustness of the presented measurements and initial validation of the dynamical similarity theorem. The quality factor of a rectangular cantilever in the continuum limit is [10]

$$
Q_{\text {cont }}=\frac{\frac{4 \rho_{\mathrm{av}} h}{\pi \rho b}+\Gamma_{r}\left(\omega_{R}\right)}{\Gamma_{i}\left(\omega_{R}\right)},
$$

where $b$ and $h$ are the cantilever width and thickness, respectively, $\Gamma\left(\omega_{R}\right)$ is the (complex-valued) hydrodynamic function [10] evaluated at the radial resonant frequency $\omega_{R}$ and the subscript $r$ and $i$ refer to its real and imaginary components, respectively. The corresponding result in the free molecular limit is $[27,32]$
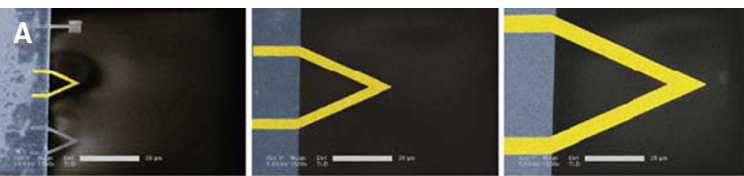

B

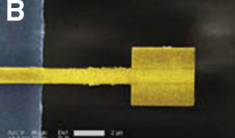

Cantilever devices $(100,300,500 \mathrm{~nm})$
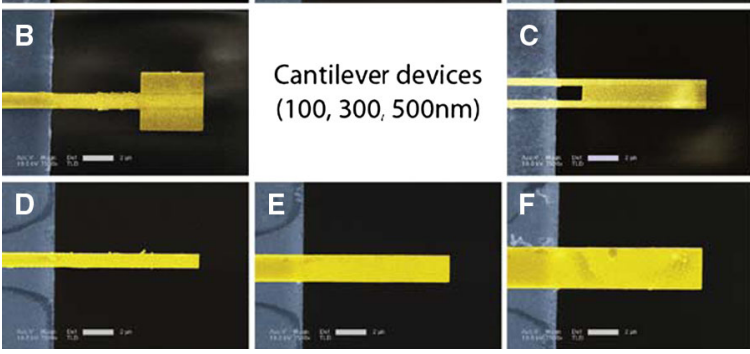

FIG. 1 (color online). SEM micrographs of cantilever devices showing plan view geometries. All devices uniformly scaled in all dimensions on 100, 300, and $500 \mathrm{~nm} \mathrm{SiN} \mathrm{films.} \mathrm{Devices} \mathrm{are} \mathrm{high-}$ lighted in yellow. Triangular devices (A) at all three size scales shown in top row. Smallest versions of all other devices fabricated from $100 \mathrm{~nm}$ thick films shown in middle and bottom rows. Scale bars for top row (device $A$ ) are $20 \mu \mathrm{m}$. Scale bars for all other devices are $2 \mu \mathrm{m}$. 


$$
Q_{\mathrm{FM}}=\frac{K(\varepsilon) \rho_{\mathrm{av}} h \omega_{\mathrm{vac}}(2 R T)^{1 / 2}}{P},
$$

where $K(\varepsilon)=\sqrt{\pi} /(4+\pi+[4-\pi] \varepsilon), \varepsilon$ is the specular reflection coefficient at the cantilever surface with $\varepsilon=1$ corresponding to pure specular reflection and $\varepsilon=0$ for pure diffuse reflection, $R$ is the specific gas constant, $T$ the absolute temperature, and $P$ the gas pressure.

Figure 2(a) presents the measured quality factors of three scaled versions of device $E$ (see Fig. 1) as a function of gas pressure; the measured intrinsic quality factor of each device is removed, as discussed [32]. At a gas pressure of $1 \mathrm{~atm}$, the quality factors of these resonators decrease as their size is uniformly reduced, which is in agreement with continuum theory, Eq. (8). In contrast, the free molecular model in Eq. (9) predicts that there will be no change in the quality factor under this uniform scaling. This is also borne out in the measurements in Fig. 2 that display a convergence of the measured quality factors of these resonators as gas pressure is reduced. Interestingly, we find that the free molecular model incorporating diffuse reflection at the solid surfaces gives best agreement with measurements; this is in accord with other studies [37]. In all cases, good agreement is observed between the measurements and predictions of the theoretical models, for both continuum and free molecular flows. These measurements on device $E$ are now used to provide an initial assessment of the dynamic similarity theorem.

Equation (7) enables the quality factor of a (unknown) nanoscale resonator at the reference density $\rho_{0}$

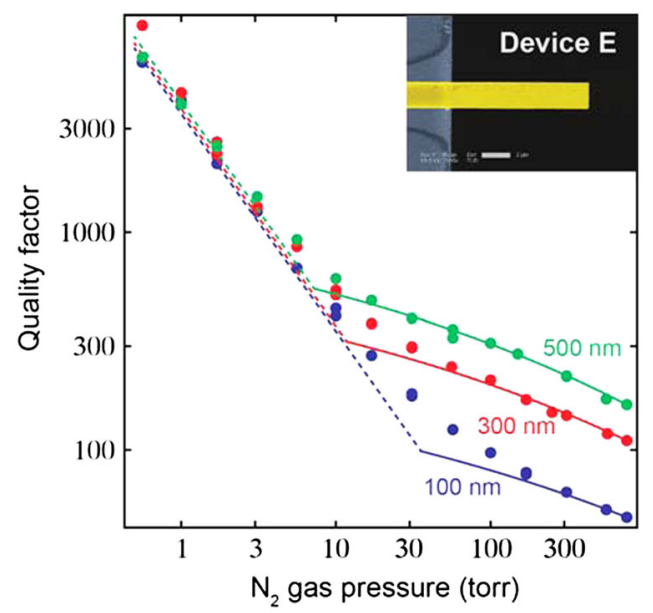

FIG. 2 (color online). Measured quality factors (due to gas) for three different sizes of device $E$ which possess identical rectangular geometries (length to width $=5$ ), as a function of gas pressure. These devices have a nominal thickness 100 (blue), 300 (red), and $500 \mathrm{~nm}$ (green), with the plan view dimensions and coatings scaled accordingly. Continuum theory, Eq. (8) (solid lines at high pressure). Free molecular theory with diffuse reflection, Eq. (9) (dashed lines at low pressure). These complementary theories are plotted up to their point of intersection, which occurs in the transition regime, $\mathrm{Kn} \sim 1$. (corresponding to a pressure $P_{0}$ ) to be determined from that of an identical scaled up (test) microscale resonator. This relationship is obtained by demanding that both resonators operate at an identical Knudsen number, Kn. In this form, the dynamic similarity theorem, Eq. (7), becomes

$$
\left.Q_{\text {scaled }}\right|_{\alpha L_{0}} ^{P_{0}}=\left.\alpha Q_{\text {test }}\right|_{L_{0}} ^{\alpha P_{0}},
$$

where $\alpha$ is the ratio of the nanoscale (scaled) resonator size to that of the microscale (test) resonator. The listed superscripts of the vertical bars in Eq. (10) are the operating gas pressures, while those in the subscripts are the resonator sizes. The symbol " 0 " is used to indicate the reference value of the indicated variable: $P_{0}$ is gas pressure corresponding to the reference density $\rho_{0}$ chosen in Eq. (7), and $L_{0}$ is the test resonator size. The material properties, mode shape, and geometry of the two resonators are identical, as is the gas used.

Physically, Eq. (10) states that "the quality factor of a nanoscale resonator immersed in a gas at pressure $P_{0}$ is $\alpha$ times that of an identical microscale resonator at the reduced pressure $\alpha P_{0}$." Importantly, this scaling law is valid for arbitrary degrees of rarefaction and encompasses the entire free molecular to continuum flow regimes, i.e., arbitrary Knudsen number. This eliminates the (problematic) need to determine the precise nature of gas-surface interactions, required for robust and accurate computational modeling $[17,18]$. These properties arise because identical materials are used for both microscale and nanoscale resonators, ensuring gas-surface interactions and the elastic material properties of their materials are also the same. Such interactions are automatically included in this theorem of dynamic similarity. As discussed, the elastic properties of nanomaterials have been found to be identical to the bulk, down to nanometer length scales [6-9].

Table I presents an experimental assessment of this theorem, giving (i) the measured quality factor of the $100 \mathrm{~nm}$ device (2nd column), and (ii) its theoretical prediction as determined from the $500 \mathrm{~nm}$ device at a reduced pressure (3rd column), see Eq. (10). Quality factors of the $500 \mathrm{~nm}$ device at the listed pressures are given in the rightmost column of Table I-note that these values are much larger than the measured quality factors for the $100 \mathrm{~nm}$ device. Strikingly, the dynamic similarity theorem yields accurate predictions for the $100 \mathrm{~nm}$ device, based on the $500 \mathrm{~nm}$ data at the pressures specified by Eq. (10). This is true for all gas pressures and over the entire range from continuum, to transition and free molecular flows, as indicated by the Knudsen numbers in Table I.

In its most general form, Eq. (7) requires determination of the function $H(\mathrm{Kn})$. This can be routinely measured by first rearranging Eq. (7): 
TABLE I. Quality factor due to surrounding gas for device $E$ of rectangular geometry (aspect ratio, length to width $=5$ ) as a function of gas pressure. Results for measurements on the $100 \mathrm{~nm}$ device (Measured); dynamic similarity theorem (DST) using $500 \mathrm{~nm}$ data. Quality factors for the $500 \mathrm{~nm}$ device are at the indicated pressures, and provided for comparison only; this is not used for the DST data on the $100 \mathrm{~nm}$ device and their Knudsen numbers are not specified. The intrinsic quality factors for the 100 and $500 \mathrm{~nm}$ devices are $Q_{\text {int }}=479.4$ and 1648 , respectively.

\begin{tabular}{lcccc}
\hline \hline & \multicolumn{3}{c}{$100 \mathrm{~nm}$ device } & \\
\cline { 2 - 3 } & \multicolumn{2}{c}{ Quality factor } & & $\begin{array}{l}\text { Quality factor } \\
\text { (500 nm device) }\end{array}$ \\
\cline { 2 - 3 } (torr) & Meas. & DST & Kn & \\
\hline 760 & 49.2 & 54.5 & 0.03 & 159 \\
310 & 63.3 & 68.2 & 0.08 & 217 \\
100 & 96.4 & 90.6 & 0.25 & 305 \\
31 & 181 & 172 & 0.82 & 400 \\
10 & 417 & 426 & 2.6 & 615 \\
3.1 & 1218 & 1205 & 8.2 & 1478 \\
1 & 3929 & 3595 & 25 & 3903 \\
\hline \hline
\end{tabular}

$$
H(\mathrm{Kn})=\left.\frac{\rho}{\rho_{0}} Q\right|_{\text {test }},
$$

where the subscript "test" refers to measurements on a single test resonator, as a function of gas pressure (density). Since the function $H(\mathrm{Kn})$ is dimensionless, it holds for all resonators of the same geometry, regardless of their size and the gas density (pressure). With this function determined, Eq. (7) can then be used to calculate the quality factor of a resonator that has been uniformly resized from the test device.

For example, to determine the quality factor (at $1 \mathrm{~atm}$ ) of a resonator of smaller size to the test resonator, we first set the reference pressure to $1 \mathrm{~atm}$. Since the quality factor of the smaller resonator is required at $1 \mathrm{~atm}$, the reference density $\rho_{0}$ and measured density $\rho$ are identical. Equation (7) then states that the quality factor of the smaller resonator is simply $Q_{\text {small }}=H\left(\mathrm{Kn}_{\text {small }}\right)$, evaluated at the Knudsen number $\mathrm{Kn}_{\text {small }}$ of the smaller resonator at $1 \mathrm{~atm}$. This provides a global picture of how the quality factor varies with resonator size, because the Knudsen number is inversely proportional to its size.

Measurements of the function $H(\mathrm{Kn})$ for devices $A, B$, $C$, and $D$ in Fig. 1 are given in Fig. 3; similar results are obtained for devices $E$ and $F$ [32]. Note that for devices of identical geometry, this dimensionless function $H(\mathrm{Kn})$ is predicted to be invariant as the device size is uniformly scaled. Furthermore, the measured quality factors of the devices differ considerably as their size is varied, as observed for device $E$ in Fig. 2; see Supplemental Material for other devices [32]. Nonetheless, the data in Fig. 3 strikingly show that the dynamic similarity theorem precisely collapses these measurements. This is despite the minimum and maximum device sizes differing by a factor
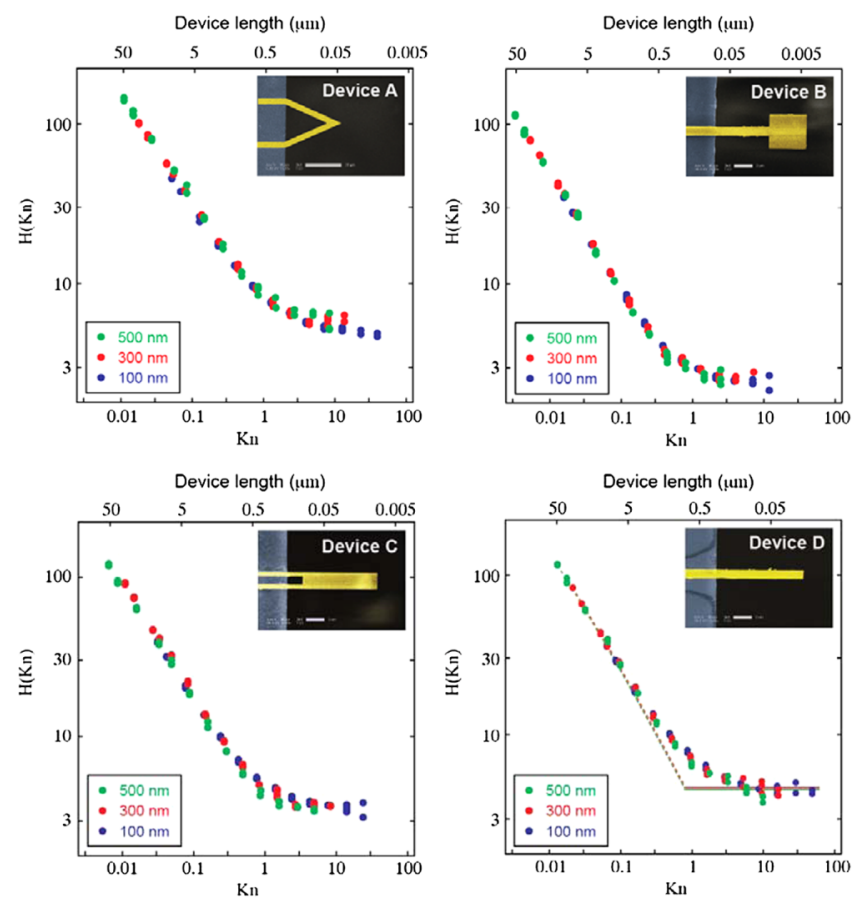

FIG. 3 (color online). Scaled quality factor $H(\mathrm{Kn})$ for cantilever devices with three different sizes: nominal thicknesses 100 (blue), 300 (red), and $500 \mathrm{~nm}$ (green). Reference gas density and pressure specified at $1 \mathrm{~atm}$. Data for devices $A, B, C$, and $D$. Other device data display similar agreement; see Supplemental Material [32]. Analytical solutions for device $D$ shown for continuum (solid lines) and free molecular flows (dashed lines), as per Fig. 2. Upper horizontal axis gives length of device for uniform reduction in size at a gas pressure of $1 \mathrm{~atm}$; setting $\rho_{0}=\rho$ in vertical axis gives the required quality factor at $1 \mathrm{~atm}$ for this size rescaling. Observed enhanced scatter in $H(\mathrm{Kn})$ at high Knudsen number is due to a finite intrinsic quality factor limiting precision at low gas pressure. Identical scales are used for the $H(\mathrm{Kn})$ and Kn axes.

of 5. The four geometries used, rectangular, triangular, paddle, and rectangular with legs, highlights the universal applicability of the dynamic similarity theorem with respect to device geometry and size variations. The data in Fig. 3 have been plotted on identical axis scales, for both the scaled quality factor and Knudsen number, to highlight the effect of resonator geometry. Figure 3, together with additional data in the Supplemental Material [32] (for all devices), shows that there are significant variations as a function of device geometry, with factors of approximately 2-5 observable. This contrasts to the high precision in which data collapse for different sized devices of identical geometry.

Data for $H(\mathrm{Kn})$ on a single test resonator can be used to determine the quality factor (at $1 \mathrm{~atm}$ ) of a resonator of arbitrary smaller size, as discussed above. Setting the reference pressure to $1 \mathrm{~atm}$ is required for this implementation. Evaluating $H(\mathrm{Kn})$ at the Knudsen number for the smaller resonator (at $1 \mathrm{~atm})$ then gives its required quality factor. 
This size scaling is indicated on the upper horizontal axis of the plots in Fig. 3, where the resized resonator length is shown. The data in Table I for the $100 \mathrm{~nm}$ device, at $1 \mathrm{~atm}$ (760 torr), are immediately evident in Fig. S8 (device $E$ ) [32]; see the upper horizontal axis at a device length of $10 \mu \mathrm{m}$.

Characterization of gas flows in the transition regime, which are generated ubiquitously by nanoscale mechanical resonators, remains a challenging problem in the physical sciences. In this Letter, we have presented a dynamic similarity theorem that facilitates such analysis at arbitrary degrees of gas rarefaction. This theorem circumvents the need for advanced computational methods of rarefied flows that can be prohibitively expensive and are seldom used. It also permits interrogation of noncontinuum gas flows at the nanoscale, through use of measurements at the more readily accessible microscale. This simple yet effective approach is expected to impact studies aimed at probing the underlying physics of gas flows and gas-structure interactions at the nanoscale, and provides a fundamental tool for design and application.

We thank D. Chi for assistance in electron beam lithography and A. Naik for helpful discussions. Experimental work at Caltech was supported by the U.S. NSF through Grant No. DBI-0821863, the NIH through Grant No. R01 GM085666-01AIZ, and a NIH Director's Pioneer Award (M. L. R.). Device fabrication was carried out in the Kavli Nanoscience Institute at Caltech. The authors gratefully acknowledge support of the Australian Research Council Grants Scheme and a Caltech's Kavli Nanoscience Institute Distinguished Visiting Professorship (J. E. S.).

*To whom all correspondence should be addressed. jsader@unimelb.edu.au

[1] G. Binnig, C. F. Quate, and C. Gerber, Phys. Rev. Lett. 56, 930 (1986).

[2] K. Jensen, K. Kim, and A. Zettl, Nat. Nanotechnol. 3, 533 (2008).

[3] H.-Y. Chiu, P. Hung, H. W. Ch. Postma, and M. Bockrath, Nano Lett. 8, 4342 (2008).

[4] N. V. Lavrik, M. J. Sepaniak, and P. G. Datskos, Rev. Sci. Instrum. 75, 2229 (2004).

[5] M. S. Hanay, S. Kelber, A. K. Naik, D. Chi, S. Hentz, E. C. Bullard, E. Colinet, L. Duraffourg, and M. L. Roukes, Nat. Nanotechnol. 7, 602 (2012).

[6] J. H. Hodak, I. Martini, and G. V. Hartland, J. Chem. Phys. 108, 9210 (1998).

[7] G. V. Hartland, Annu. Rev. Phys. Chem. 57, 403 (2006).

[8] M. Pelton, J. E. Sader, J. Burgin, M. Liu, P. Guyot-Sionnest, and D. Gosztola, Nat. Nanotechnol. 4, 492 (2009).

[9] V. Juvé, A. Crut, P. Maioli, M. Pellarin, M. Broyer, N. Del Fatti, and F. Valleé, Nano Lett. 10, 1853 (2010).

[10] J. E. Sader, J. Appl. Phys. 84, 64 (1998).
[11] M. Li, H. X. Tang, and M. L. Roukes, Nat. Nanotechnol. 2, 114 (2007).

[12] K. L. Ekinci, D. M. Karabacak, and V. Yakhot, Phys. Rev. Lett. 101, 264501 (2008).

[13] O. Svitelskiy, V. Sauer, N. Liu, K.-M. Cheng, E. Finley, M. R. Freeman, and W. K. Hiebert, Phys. Rev. Lett. 103, 244501 (2009).

[14] C. Lissandrello, V. Yakhot, and K. L. Ekinci, Phys. Rev. Lett. 108, 084501 (2012).

[15] T. M. Squires and S. R. Quake, Rev. Mod. Phys. 77, 977 (2005).

[16] E. Lauga, M. P. Brenner, and H. A. Stone, in Handbook of Experimental Fluid Dynamics, edited by J. Foss, C. Tropea, and A. Yarin (Springer, New York, 2005).

[17] C. Cercignani, Slow Rarefied Flows: Theory and Application to Micro-Electro-Mechanical Systems (Birkhäuser Verlag, Basel, 2000).

[18] Y. Sone, Molecular Gas Dynamics: Theory, Techniques, and Applications (Birkhäuser Verlag, Basel, 2007).

[19] W. G. Vincenti and C. H. Kruger, Jr., Introduction to Physical Gas Dynamics (Wiley, New York, 1965).

[20] R. J. Clarke, O. E. Jensen, J. Billingham, and P. M. Williams, Proc. R. Soc. A 462, 913 (2006).

[21] WolframAlpha (http://www.wolframalpha.com).

[22] G. Karniadakis, A. Beskok, and N. Aluru, Microflows and Nanoflows: Fundamentals and Simulation (Springer, New York, 2005). These ranges are loosely defined and used as a guide only.

[23] J. Nassios and J.E. Sader, J. Fluid Mech. 708, 197 (2012).

[24] L. L. Baker and N. G. Hadjiconstantinou, Int. J. Numer. Methods Fluids 58, 381 (2008).

[25] G. A. Radtke, N. H. Hadjiconstantinou, and W. Wagner, Phys. Fluids 23, 030606 (2011).

[26] J. M. Reese, M. A. Gallis, and D. A. Lockery, Phil. Trans. R. Soc. A 361, 2967 (2003).

[27] R. A. Bidkar, R. C. Tung, A. A. Alexeenko, H. Sumali, and A. Raman, Appl. Phys. Lett. 94, 163117 (2009).

[28] P. W. Bridgman, Dimensional Analysis (Yale University, New Haven, CT, 1931).

[29] K. L. Ekinci and M. L. Roukes, Rev. Sci. Instrum. 76, 061101 (2005).

[30] J. E. Sader, J. Pacifico, C. P. Green, and P. Mulvaney, J. Appl. Phys. 97, 124903 (2005).

[31] J. E. Sader, J. A. Sanelli, B. D. Adamson, J. P. Monty, X. Wei, S. A. Crawford, J. R. Friend, I. Marusic, P. Mulvaney, and E. J. Bieske, Rev. Sci. Instrum. 83, 103705 (2012).

[32] See Supplemental Material at http://link.aps.org/ supplemental/10.1103/PhysRevLett.112.015501 for derivation of theoretical results, description of fabrication, methods and further data.

[33] This parameter is often referred to under different names, e.g., Reynolds, inverse Stokes, or the Womersley number.

[34] Finite mean free path reduces this difference [11].

[35] The coefficient $a$ depends on the material, geometry, and mode shape. It is not required in this analysis.

[36] W. E. Newell, Science 161, 1320 (1968).

[37] G. A. Bird, Molecular Gas Dynamics and the Direct Simulation of Gas Flows (Clarendon, Oxford, 1994). 